\title{
The cognitive and behavioral effects of meningioma lesions involving the ventromedial prefrontal cortex
}

\author{
Taylor J. Abel, MD, ${ }^{1}$ Kenneth Manzel, BS, ${ }^{2}$ Joel Bruss, BA, ${ }^{2}$ Amy M. Belfi, PhD, ${ }^{2}$ \\ Matthew A. Howard III, MD, ${ }^{1}$ and Daniel Tranel, PhD',3 \\ Departments of ${ }^{1}$ Neurosurgery, ${ }^{2}$ Neurology, and ${ }^{3}$ Psychology, University of lowa, lowa City, lowa
}

\begin{abstract}
OBJECTIVE Anterior skull base meningiomas are frequently associated with changes in personality and behavior. Although such meningiomas often damage the ventromedial prefrontal cortex (vmPFC), which is important for higher cognition, the cognitive and behavioral effects of these meningiomas remain poorly understood. Using detailed neuropsychological assessments in a large series of patients, this study examined the cognitive and behavioral effects of meningioma lesions involving the vmPFC.
\end{abstract}

METHODS The authors reviewed neuropsychology and lesion mapping records of 70 patients who underwent resection of meningiomas. The patients were drawn from the Neurological Patient Registry at the University of lowa. Patients were sorted into 2 groups: those with lesions involving the vmPFC and those with lesions that did not involve the vmPFC. Neuropsychological data pertaining to a comprehensive array of cognitive and behavioral domains were available preoperatively in 20 patients and postoperatively in all 70 patients.

RESULTS No change occurred in basic cognitive functions (e.g., attention, perception, memory, construction and motor performance, language, or executive functions) from the preoperative to postoperative epochs for the VmPFC and non-vmPFC groups. There was a significant decline in the behavioral domain, specifically adaptive function, for both the vmPFC and non-vmPFC groups, and this decline was more pronounced for the vmPFC group. Additionally, postoperative data indicated that the vmPFC group had a specific deficit in value-based decision making, as evidenced by poor performance on the lowa Gambling Task, compared with the non-vmPFC group. The vmPFC and non-vmPFC groups did not differ postoperatively on other cognitive measures, including intellect, memory, language, and perception.

CONCLUSIONS Lesions of the VmPFC resulting from meningiomas are associated with specific deficits in adaptive function and value-based decision making. Meningioma patients showed a decline in adaptive function postoperatively, and this decline was especially notable in patients with vmPFC region meningiomas. Early detection and resection of meningiomas of the anterior skull base (involving the gyrus rectus) may prevent these deficits.

http://thejns.org/doi/abs/10.3171/2015.5.JNS142788

KEY WORDS adaptive function; brain tumor; decision making; lowa Gambling Task; neuropsychology; oncology; personality change

$\mathrm{A}$ NTERIOR skull base meningiomas are frequently associated with changes in personality and behavior. ${ }^{10,14,16}$ Interestingly, despite the frequency of meningiomas affecting personality and behavior, large neuropsychological studies documenting alterations in the cognitive (e.g., perception, memory, executive, and language functions) and behavioral domains (e.g., personal adjustment and emotional function) in these patients are rare. Case reports have been published describing personality changes in patients with meningioma ${ }^{21-23}$ but larger studies specifically describing personality change in an- terior skull base meningioma patients are lacking. Some neuropsychological studies have described basic cognitive outcomes in patients with anterior skull base meningioma. ${ }^{8,18,26}$ These studies report deficits in cognitive functioning, specifically with executive function, working memory, processing capacity, and motor speed. Documenting outcomes in the behavioral domain in addition to the cognitive domain is crucial, because deficits in the behavioral domain have close relevance to the personality changes frequently associated with anterior skull base meningiomas. 
Anterior skull base meningiomas often involve the ventromedial prefrontal cortex (vmPFC), which is implicated in various higher cognitive functions (including valuebased decision-making ${ }^{5}$ and moral judgments ${ }^{17}$ ) and also in emotional regulation ${ }^{1}$ and personality. ${ }^{2,3}$ Interestingly, although anterior skull base meningiomas frequently involve (and lesion) the vmPFC, the cognitive and behavioral manifestations of anterior skull base meningiomas remain poorly understood. ${ }^{26}$ Several authors have reported minimal change in cognition following resection for meningiomas. ${ }^{18,26}$ However, despite no significant measureable difference in cognitive or behavioral functioning after surgery, many patients do not return to their premorbid level of functioning. ${ }^{18}$ Such outcomes suggest that meningioma lesions, albeit not entirely caused by surgery itself, can lead to significant morbidity after treatment. The purpose of the current study was to characterize the cognitive and behavioral effects of meningioma lesions involving the vmPFC. We hypothesized that meningiomas involving the vmPFC would be associated with a specific decline in adaptive functioning, even when other cognitive functions are spared. We addressed this hypothesis by taking advantage of a unique opportunity afforded by the Iowa Neurological Patient Registry, which contains a large number of patients $(n=70)$ who underwent resection of meningiomas. This dataset allowed us to incorporate two important design features: 1) for patients with pre- and postsurgical neuropsychological assessments $(n=20)$, we could examine the effects of surgery per se; and 2) we could contrast patients with vmPFC meningioma lesions $(n=23)$ with patients with non-vmPFC meningioma lesions $(\mathrm{n}=47)$.

\section{Methods}

\section{Patient Population}

The participants in this study $(\mathrm{n}=70)$ were drawn from the Neurological Patient Registry in the Division of Cognitive Neuroscience at the University of Iowa. All participants had stable, focal lesions following meningioma resection. Exclusion criteria included dementia, concomitant neurological disease (e.g., evidence of cerebral infarction), premorbid psychiatric disease, developmental abnormalities, and alcohol or drug abuse. All participants provided informed consent in accordance with the Human Subjects Committee of the University of Iowa. In connection with their enrollment in the Patient Registry, patients were extensively characterized neuropsychologically and neuroanatomically, using standard protocols of the Benton Neuropsychology Laboratory ${ }^{25}$ and the Human Neuroanatomy and Neuroimaging Laboratory. ${ }^{11}$ All data were collected in the chronic epoch of recovery (i.e., at least 3 months postoperatively). The average ( \pm SD) age of the participants was $62.1 \pm 12.6$ years, and the average years of education was $13.5 \pm 2.5$ years. Patients were categorized into 2 groups: those that have lesions to the vmPFC and those that do not (Fig. 1). Patients were placed in the vmPFC group when their lesion included either the left or right gryus rectus. The demographics for participants in the vmPFC group ( $n=23 ; 7$ men, 16 women) and the nonvmPFC group ( $\mathrm{n}=47 ; 18$ men, 29 women) are listed in Table 1. Independent sample t-tests revealed that participants in the vmPFC group were older at the time of testing postoperatively than those in the non-vmPFC group ( $\mathrm{t}$ [68] $=3.2, p=0.002)$, and had longer postoperative times since their resection ( $[68]=2.6, p=0.01)$. For patients in the vmPFC group, subjective personality disturbance was the most common presenting complaint and occurred in $74 \%$ of patients. In the non-vmPFC group, headache was the most common presenting complaint, occurring in $26 \%$ of patients (Table 2).

A subset of patients $(n=20)$ was also studied preoperatively. Ten patients with meningioma involving the vmPFC ( 3 men, 7 women; mean preoperative age $58.9 \pm$ 11.7 years old, postsurgery $68.4 \pm 11.2$ years old; mean years of education $11.9 \pm 2.7$ years) were compared with 10 patients with meningioma outside the vmPFC (6 men, 4 women; mean preoperative age $57.0 \pm 15.4$ years old, postsurgery $61.3 \pm 15.0$ years old; mean years of education $13.4 \pm 2.5$ years). There were no significant differences between the 2 groups with respect to years of education or age at the time of testing both before and after surgery.

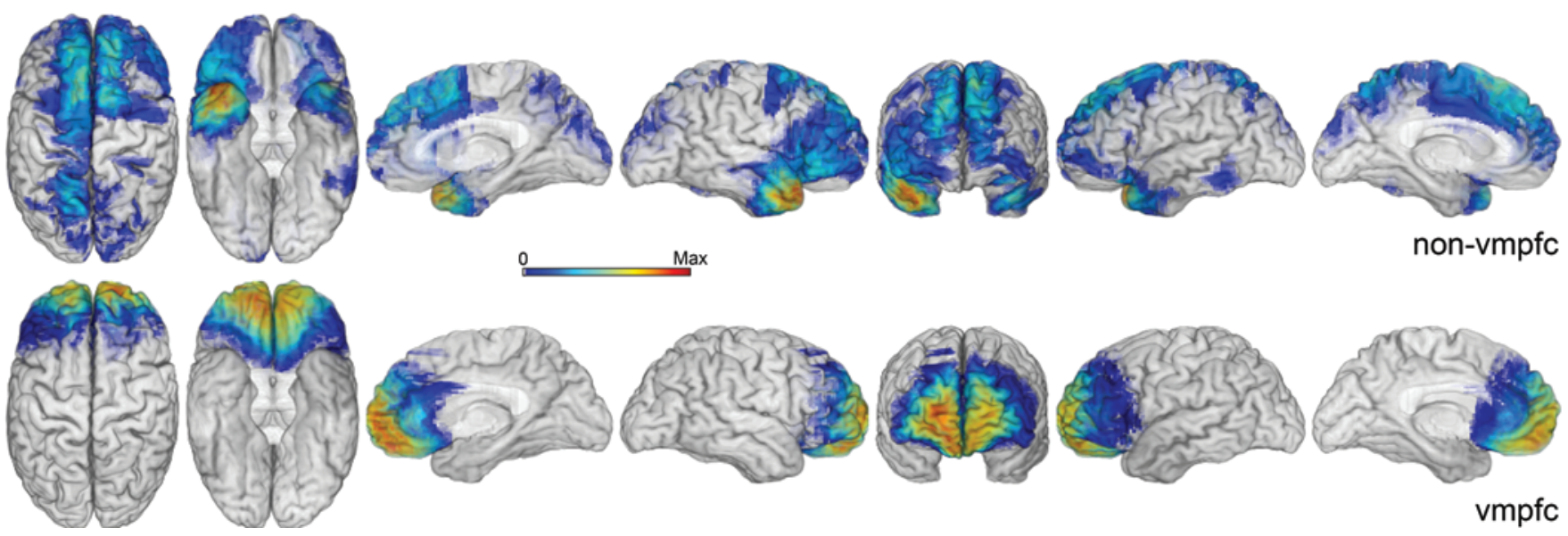

FIG. 1. Lesion maps for non-vmPFC (upper row, $n=32$ ) and vmPFC (lower row, $n=19$ ) patient groups. Color scale represents the degree of lesion overlap across patients in each group with red (the right side of color scale) representing maximum lesion overlap (non-vmPFC maximum overlap $=8$, vmPFC maximum overlap $=18$ ). 
TABLE 1. Demographics of all participants

\begin{tabular}{lccc}
\hline \multicolumn{1}{c}{ Variable } & Age & Yrs of Education & Chronicity (yrs) \\
\hline Mean \pm SD & $62.1 \pm 12.6$ & $13.5 \pm 2.5$ & $5.7 \pm 6.3$ \\
\hline Range & $30-86$ & $8-20$ & $0.6-38.5$ \\
\hline vmPFC $(n=23)$ & & & \\
\hline \multicolumn{1}{c}{ Mean \pm SD } & $68.6 \pm 9.7^{*}$ & $13.2 \pm 2.8$ & $8.4 \pm 8.8^{*}$ \\
\hline non-vmPFC & & & \\
\hline Mean \pm SD & $58.9 \pm 12.7$ & $13.7 \pm 2.3$ & $4.3 \pm 4.0$ \\
\hline
\end{tabular}

Chronicity $=$ length of time since surgery.

* Indicates statistical significance.

\section{Lesion Analysis}

Lesion mapping procedures were performed as previously described. ${ }^{6,711,13}$ To analyze the placement of lesions and the overlap of lesions in specific brain areas in a group this size, it is necessary to place all lesions in a common space. Lesions were identified by hypointensity on a T1weighted MRI sequence or hypodensity on CT. The lesion contour from the lesioned brains is manually warped into a normal template brain, taking into account gyral and sulcal landmarks, using the following steps: 1) a normal brain, the template brain, is reconstructed in 3 dimensions from thin contiguous MR slices using Brainvox (version 3.14); 2) all major sulci are identified and color-coded in this template brain as well as in the lesion brain; 3) the template brain volume is resliced so as to match the MR slices (or CT slices) of the lesioned brain; 4) the slices in the template brain are matched in orientation and thickness to the lesioned brain, taking into consideration the intersection of the slices with the color-coded sulci (a good match can be assured both by inspection of the 2D images as well as by the positioning of the slices seen in the $3 \mathrm{D}$ images); 5) once the matching slices have been obtained, the lesion contour on each slice is manually transferred onto the template brain, taking into consideration the distance of the lesion contour to the identifiable landmarks, such as gyri and subcortical structures; 6) the collection of transferred traces defines a volume that can be co-rendered with the template brain; and 7) the volumes of several lesions so transferred into the template brain intersect in space and create a complex volume, which can also be co-rendered with the template brain. The overlaps of lesions in this volume, calculated by the sum of lesions overlapping on any single voxel, can be color-coded. Thus, the final overlap of subject lesions can be analyzed in the template brain for areas corresponding to maximal overlaps.

Given the robust literature demonstrating a role for the vmPFC cortex in personality and emotional regulation, ${ }^{1-3}$ patients were stratified into 2 groups based on whether their lesion associated with their meningioma involved the vmPFC as described above. Lesion size analysis was performed for all patients with appropriate neuroimaging data (51 patients; 19 from the vmPFC group and 32 from the non-vmPFC group). For the patients that had a defined lesion mask, the volume of the mask was calculated using fslstats (version 5.0.2.2), a tool provided in FSL's fslutils. ${ }^{15}$ All lesion masks were first binarized so that voxels containing a lesion had a value of 1 and voxels outside the lesion had a value of zero. The resultant mask was run
TABLE 2. Presenting complaint by lesion

\begin{tabular}{|c|c|}
\hline Complaint & Value (\%) \\
\hline \multicolumn{2}{|l|}{$\operatorname{vmPFC}(n=23)$} \\
\hline Personality disturbance & $17(74)$ \\
\hline Anosmia & $6(26)$ \\
\hline Visual symptoms & $5(22)$ \\
\hline Memory impairment & $3(13)$ \\
\hline Confusion & $2(9)$ \\
\hline Headache & $2(9)$ \\
\hline Seizure & $2(9)$ \\
\hline Imbalance & $1(4)$ \\
\hline Incidental & $1(4)$ \\
\hline \multicolumn{2}{|l|}{ non-vmPFC $(n=47)$} \\
\hline Headache & $12(26)$ \\
\hline Visual symptoms & $9(19)$ \\
\hline Personality disturbance & $8(17)$ \\
\hline Seizure & $7(15)$ \\
\hline Motor disturbance & $5(11)$ \\
\hline Language impairment & $5(11)$ \\
\hline Imbalance & $4(9)$ \\
\hline Memory impairment & $4(9)$ \\
\hline Gait abnormality & $2(4)$ \\
\hline Diplopia & $2(4)$ \\
\hline Dizziness & $2(4)$ \\
\hline Confusion & $2(4)$ \\
\hline Vertigo & $1(2)$ \\
\hline Incidental & $1(2)$ \\
\hline Sensory disturbance & $3(6)$ \\
\hline Hoarse voice & $1(2)$ \\
\hline Spatial disorientation & $1(2)$ \\
\hline Depression & $1(2)$ \\
\hline Eye swelling & $1(2)$ \\
\hline Syncope & $1(2)$ \\
\hline Incontinence & $1(2)$ \\
\hline Ataxia & $1(2)$ \\
\hline Proptosis & $1(2)$ \\
\hline
\end{tabular}

through fslstats, and the output volume $\left(\mathrm{mm}^{3}\right)$ for nonzero voxels was calculated.

\section{Neuropsychological Testing}

Participants were assessed according to standard protocols of the Benton Neuropsychology Laboratory. ${ }^{25}$ This entails standardized assessment of classically described cognitive domains: ${ }^{20}$ orientation and attention, perception, memory, verbal functions and language skills, construction and motor skills, concept formation and reasoning, and executive functions (Table 3). Behavioral domains assessed included personal adjustment and emotional function, and adaptive functions (Table 3). Scores in domains of personal adjustment and emotional function, and adaptive functions, were determined by a trained neuropsychologist who was blind to the objectives and hypotheses 
TABLE 3. Neuropsychological tests in each cognitive and behavioral domain

\begin{tabular}{|c|c|}
\hline Orientation \& Attention & Concept Formation \& Reasoning \\
\hline Orientation & WAIS-similarities \\
\hline Digit span & WAIS-comprehension \\
\hline Letter-number sequencing & WAIS-arithmetic \\
\hline Spatial span & WAIS-matrix reasoning \\
\hline Sentence repetition & WAIS-picture completion \\
\hline Stroop & WAIS-picture arrangement \\
\hline Coding & WAIS-figure weights \\
\hline Symbol search & WAIS-visual puzzles \\
\hline TMT A & WRAT-arithmetic \\
\hline Perception & WCST-categories \& perseverative errors \\
\hline JoLO & Executive functions \\
\hline Benton faces & IGT \\
\hline Hooper & COWA \\
\hline Memory & Category fluency \\
\hline AVLT-5' \& AVLT-30' & TMT B \\
\hline CFT-recall & Personal adjustment \& emotional function \\
\hline BVRT-correct & $\mathrm{BDI}$ \\
\hline WMS-indices & $\mathrm{BAI}$ \\
\hline Verbal functions \& language skills & ISPC \\
\hline BNT & MMPI \\
\hline WAIS vocabulary & Adaptive functions \\
\hline WAIS information & Blinded chart review of outcome (e.g., employment, independence, self-care) \\
\hline \multicolumn{2}{|l|}{ Token test } \\
\hline \multicolumn{2}{|l|}{ WRAT-reading } \\
\hline \multicolumn{2}{|l|}{ WRAT-spelling } \\
\hline \multicolumn{2}{|l|}{ ICRT } \\
\hline \multicolumn{2}{|l|}{ Construction \& motor performance } \\
\hline \multicolumn{2}{|l|}{ CFT-copy } \\
\hline \multicolumn{2}{|l|}{ WAIS-block design } \\
\hline \multicolumn{2}{|l|}{ WAIS-object assembly } \\
\hline Grooved pegboard & \\
\hline
\end{tabular}

AVLT-5', -30' = Auditory Verbal Learning Test (5' = 5 minute delay, 30' = minute delay); BAI = Beck Anxiety Inventory; BDI = Beck Depression Inventory; BNT = Boston Naming Test; BVRT = Benton Visual Retention Test; $C F T=$ Complex Figure Test; COWA = Controlled Oral Word Association; ICRT = lowa-Chapman Reading Test; ISPC = lowa Scale of Personality Change; JoLO = Judgment of Line Orientation; MMPI = Minnesota Multiphasic Personality Inventory; TMT A, B = Trail Making Test, Parts A \& B; WAIS = Wechsler Adult Intelligence Scale; WCST = Wisconsin Card Sorting Test; WMS = Weschler Memory Scale; WRAT = Wide Range Achievement Test.

of the study. To assess personal adjustment and emotional function, standardized self-report measures (e.g., Minnesota Multiphasic Personality Inventory, Beck Depression Inventory) and collateral ratings (e.g., Iowa Scales of Personality Change) were reviewed. To assess adaptive functions, each patient's history and clinical notes were reviewed with regard to employment, independence, and self-care. Using findings from this chart review, each patient's personal adjustment and emotional function, and adaptive functions, were assigned a rating of impairment $(0=$ no impairment, $1=$ mild impairment, $2=$ moderate impairment, and $3=$ severe impairment) by the blinded neuropsychologist who performed the chart review.

To assess real-life decision making, a subset of patients were tested using the Iowa Gambling Task (IGT)., ${ }^{4,5}$ The IGT is a neuropsychological task designed to evaluate and quantify deficits in real-life decision-making during conditions of immediate and delayed reward and punishment. ${ }^{4,5}$ In the task, patients are given \$2000 in virtual money and told to play a game to maximize the amount of money they make. Four card decks are presented: A, B, C, and $\mathrm{D}$, with $\mathrm{A}$ and $\mathrm{B}$ associated with $\$ 100$ in immediate reward and $\mathrm{C}$ and $\mathrm{D}$ associated with $\$ 50$ in immediate reward. Unpredictably, decks A and B are associated with a large penalty, while $C$ and $D$ have smaller penalties. Thus, patients who pick mostly from $\mathrm{A}$ and $\mathrm{B}$ will incur a net loss, while patients who pick mostly from $\mathrm{C}$ and $\mathrm{D}$ will incur a net gain (despite the lower "up front" reward in C and D). In the task, there are 100 trials, analyzed by blocks of 20 (blocks 1-5). During the task, patients are not told how many trials they will complete. Patients with vmPFC damage are known to have deficits on the IGT. ${ }^{5}$ 


\section{Statistical Analysis}

All raw scores were converted to z-scores in accordance with available normative data. ${ }^{20}$ Normalized neuropsychological test data were averaged within each of the aforementioned cognitive domains to obtain a composite z-score.

\section{Results}

\section{Comparison of Pre- and Postoperative Cognitive and Adaptive Functions}

Independent-sample t-tests (Bonferroni corrected) were performed to assess group differences (vmPFC vs nonvmPFC) in the change scores from pre- to postoperative testing in the 20 patients with both pre- and postoperative data. These revealed no significant differences between the 2 groups with respect to any of the cognitive domains (Table 4). Similar results were found when looking at individual subtests making up the domains (Table 4). Both groups showed declines in depressive symptomatology (less depression) postoperatively, as measured by the Beck Depression Inventory, but again no significant differences were found between the 2 groups.

The behavioral domain of adaptive function was assessed in all patients $(n=70)$ before and after surgery. A $2 \times 2$ ANOVA was performed to assess differences in adaptive function in the pre- and postoperative epochs as a function of lesion location, where group (vmPFC vs nonvmPFC) and epoch (preoperative vs postoperative) were the 2 factors in the ANOVA. This analysis yielded a significant interaction between group and epoch $(\mathrm{F}[1,68]=$ $4.50, p=0.04)$, reflecting that the magnitude of postoperative adaptive function decline was greater for the vmPFC group. Both main effects were also significant, reflecting that adaptive function scores in the vmPFC group were lower than those in the non-vmPFC group both preoperatively and postoperatively $(\mathrm{F}[1,68]=17.42, \mathrm{p}<0.001)$, and that there was a decline in adaptive function from the preto the postoperative epochs for both the vmPFC and nonvmPFC groups $(F[1,68]=41.56, p<0.001)$. These data are shown in Fig. 2.

\section{Postoperative Cognitive and Behavioral Domain Performance}

Comparison of postoperative performances in each cognitive domain, and individual tests comprising the domains, are summarized in Tables 5 and 6 , respectively. Independent sample t-tests revealed no significant differences $(p>0.05)$ between the vmPFC and non-vmPFC groups with respect to any cognitive domain (Table 5) or individual test (Table 6). Given the variability of age and length of time since surgery (chronicity) parameters, a regression analysis was performed to account for age and chronicity. This yielded the same result, i.e., no significant difference between the vmPFC and non-vmPFC groups with regard to postoperative impairment in any of the cognitive domains.

In contrast to the cognitive domains, a significant difference existed between the 2 groups with respect to the behavioral domain of adaptive functions. Independent samples t-tests revealed that participants in the vmPFC
TABLE 4. Comparison of patients tested pre- and postoperatively*

\begin{tabular}{|c|c|c|}
\hline Variable & $\begin{array}{c}\text { vmPFC } \\
\text { Change } \\
\text { Score } \pm \text { SD }\end{array}$ & $\begin{array}{c}\text { Non-vmPFC } \\
\text { Change } \\
\text { Score } \pm \text { SD }\end{array}$ \\
\hline \multicolumn{3}{|l|}{ Cognitive domain } \\
\hline Attention & $0.8 \pm 0.9$ & $0.7 \pm 1.1$ \\
\hline Perception & $0.7 \pm 1.4$ & $-0.1 \pm 1.5$ \\
\hline Memory & $0.3 \pm 0.8$ & $0.7 \pm 1.4$ \\
\hline Construction \& motor performance & $0.5 \pm 1.3$ & $0.3 \pm 1.4$ \\
\hline Verbal functions \& language skills & $-0.3 \pm 0.6$ & $0.7 \pm 3.0$ \\
\hline Concept formation \& reasoning & $0.2 \pm 0.9$ & $0.3 \pm 0.8$ \\
\hline Executive functions & $1.3 \pm 2.8$ & $1.8 \pm 0.9$ \\
\hline \multicolumn{3}{|l|}{ Individual tests } \\
\hline WAIS verbal IQ & $-0.1 \pm 0.6$ & $0.1 \pm 0.1$ \\
\hline WAIS similarities & $-0.1 \pm 0.8$ & $-0.1 \pm 0.6$ \\
\hline WAIS digit span & $0.3 \pm 0.6$ & $-0.1 \pm 0.9$ \\
\hline WAIS arithmetic & $-0.4 \pm 0.9$ & $0.1 \pm 0.4$ \\
\hline WAIS block design & $0.1 \pm 0.7$ & $-0.1 \pm 0.6$ \\
\hline WAIS coding & $1.2 \pm 1.4$ & $0.1 \pm 0.6$ \\
\hline BVRT correct & $0.5 \pm 1.3$ & $0.6 \pm 1.6$ \\
\hline BVRT errors & $0.4 \pm 1.4$ & $0.8 \pm 1.5$ \\
\hline AVLT trial 5 & $0.6 \pm 0.9$ & $-0.1 \pm 2.2$ \\
\hline AVLT 30' delay & $-0.1 \pm 1.2$ & $0.3 \pm 1.4$ \\
\hline CFT copy & $1.0 \pm 1.0$ & $0.9 \pm 2.1$ \\
\hline CFT recall & $2.1 \pm 1.8$ & $1.1 \pm 1.1$ \\
\hline BNT & $0.6 \pm 0.4$ & $2.8 \pm 5.7$ \\
\hline COWA & $1.6 \pm 2.1$ & $0.4 \pm 0.7$ \\
\hline Facial Recognition Test & $0.1 \pm 1.0$ & $-0.4 \pm 1.3$ \\
\hline TMT A & $3.0 \pm 2.3$ & $1.9 \pm 2.3$ \\
\hline TMT B & $2.6 \pm 2.1$ & $2.5 \pm 4.5$ \\
\hline Pegboard dominant & $2.0 \pm 2.6$ & $-1.7 \pm 1.6$ \\
\hline Pegboard nondominant & $2.7 \pm 3.2$ & $-0.3 \pm 2.0$ \\
\hline WCST categories & $1.6 \pm 1.3$ & $0.3 \pm 2.3$ \\
\hline WCST perseverative errors & $3.9 \pm 4.1$ & $0.3 \pm 2.4$ \\
\hline $\mathrm{BPI}^{*}$ & $-8.4 \pm 6.5$ & $-12.6 \pm 5.6$ \\
\hline
\end{tabular}

\footnotetext{
* Change scores reflect the change in composite z-scores from pre- to postoperative testing. A positive value indicates relative improvement, whereas a negative value indicates a relative decline. Beck Depression Inventory is a change in the raw score from pre- to postoperative testing. Negative values reflect decline in number of depressive symptoms endorsed.
}

group had a significantly higher rate of impairment than did participants in the non-vmPFC group (t [68] $=5.8$, $\mathrm{p}$ $<0.001$; Fig. 3). No significant differences were found between the groups with respect to emotional function. Similar to the cognitive domains, a regression analysis was performed to account for variability in age and chronicity between the vmPFC and non-vmPFC groups and when accounting for these variables, the vmPFC group still had a significantly higher rate of adaptive function impairment than the non-vmPFC group ( $<<0.001)$.

\section{lowa Gambling Task Performance}

A subset of the participants was administered the IGT 


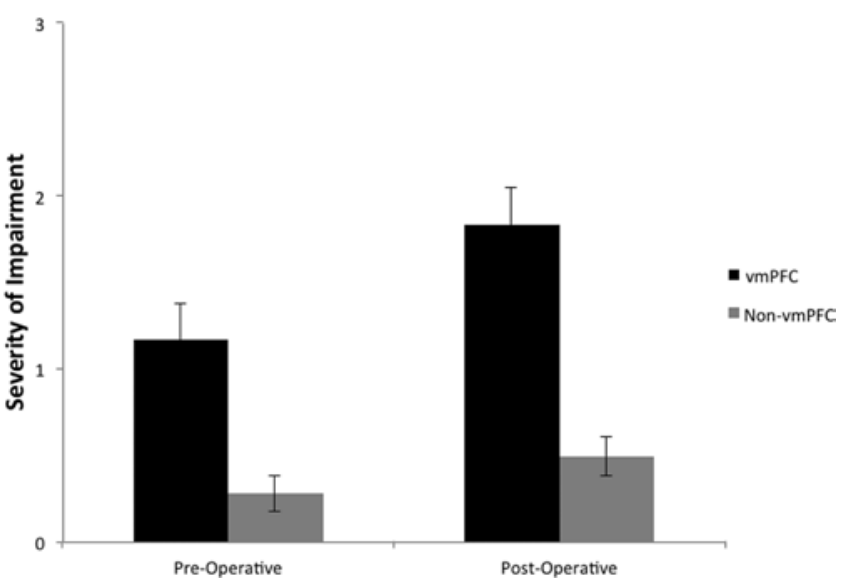

FIG. 2. Pre- and postoperative average scores for adaptive functioning in the vmPFC and non-vmPFC groups. Numbers reflect average rating from 0 to $3(0=$ no impairment; 1 = mild impairment; 2 = moderate impairment; 3 = severe impairment).

(vmPFC: $\mathrm{n}=11$; non-vmPFC: $\mathrm{n}=15$ ). Participants in the vmPFC group performed significantly worse than nonvmPFC participants did during Block 5 (the final 20 card selections) of the task ( $\mathrm{t}[24]=-2.2, \mathrm{p}=0.03)$. A trend toward significance was noted when comparing the 2 groups over the entire task of 100 selections ( $[24]=-1.9, \mathrm{p}=$ 0.08; Fig. 4).

Chi-square analysis of task performance revealed that a significantly higher proportion of vmPFC participants had impaired performances both on Block $5\left(\chi^{2}[1, \mathrm{n}=26]=\right.$ $5.38, \mathrm{p}=0.02)$ and the overall task $\left(\chi^{2}[1, \mathrm{n}=26]=9.67\right.$, $\mathrm{p}=0.002)$. The 2 groups did not differ significantly with respect to age at the time of testing ( $\mathrm{vmPFC}$ mean $=67.1$ \pm 6.8 years; non-vmPFC mean $=58.1 \pm 13.8$ years), lesion chronicity (vmPFC mean $=12.5 \pm 11.0$ years; non-vmPFC mean $=7.0 \pm 3.4$ years), or years of education ( $(\mathrm{vmPFC}$ mean $=14.9 \pm 2.5$ years; non-vmPFC mean $=14.0 \pm 2.6$ years).

Further analysis showed significant correlations between adaptive functions and performance on the IGT. Greater impairment in adaptive functions was significantly correlated with worse performance on the IGT both on Block $5(\mathrm{r}=-0.63, \mathrm{p}=0.001)$ and the overall task $(\mathrm{r}=$ $-0.57, \mathrm{p}=0.002)$. Of the $12 \mathrm{vmPFC}$ participants, 7 were impaired on Block 5 of the IGT, and all had ratings of moderately or severely impaired adaptive functions. Five

TABLE 5. Comparison of cognitive domains in all patients

\begin{tabular}{lcc}
\hline \multicolumn{1}{c}{ Cognitive Domain } & $\begin{array}{c}\text { vmPFC } \\
\text { Average } \\
\text { z-Score } \pm \text { SD }\end{array}$ & $\begin{array}{c}\text { Non-vmPFC } \\
\text { Average } \\
\text { z-Score } \pm \text { SD }\end{array}$ \\
\hline Attention & $0.0 \pm 1.1$ & $0.0 \pm 0.8$ \\
\hline Perception & $0.2 \pm 1.1$ & $-0.2 \pm 0.9$ \\
\hline Memory & $0.2 \pm 1.3$ & $0.0 \pm 1.0$ \\
\hline Construction \& motor performance & $0.3 \pm 0.8$ & $-0.1 \pm 1.4$ \\
\hline Verbal function \& language skills & $0.1 \pm 0.8$ & $0.0 \pm 0.7$ \\
\hline Concept formation \& reasoning & $0.3 \pm 0.7$ & $0.1 \pm 0.7$ \\
\hline Executive functions & $-0.4 \pm 1.6$ & $-0.3 \pm 1.2$ \\
\hline
\end{tabular}

TABLE 6. Comparison of individual test scores for vmPFC and non-vmPFC groups in the postoperative epoch

\begin{tabular}{lcc}
\hline \multicolumn{1}{c}{ Individual Test } & $\begin{array}{c}\text { vmPFC } \\
\text { z-Score } \pm \text { SD }\end{array}$ & $\begin{array}{c}\text { Non-vmPFC } \\
\text { z-Score } \pm \text { SD }\end{array}$ \\
\hline WAIS verbal IQ & $0.4 \pm 0.9$ & $0.0 \pm 0.9$ \\
\hline WAIS performance IQ & $0.3 \pm 0.9$ & $0.1 \pm 0.9$ \\
\hline WAIS full-scale IQ & $0.4 \pm 1.0$ & $0.1 \pm 0.9$ \\
\hline WAIS similarities & $0.4 \pm 0.9$ & $0.1 \pm 0.9$ \\
\hline WAIS information & $0.2 \pm 1.1$ & $-0.1 \pm 0.9$ \\
\hline WAIS digit span & $0.2 \pm 0.8$ & $-0.3 \pm 0.9$ \\
\hline WAIS arithmetic & $0.4 \pm 1.0$ & $0.0 \pm 1.0$ \\
\hline WAIS block design & $0.5 \pm 0.7$ & $0.0 \pm 0.9$ \\
\hline WAIS coding & $0.1 \pm 1.0$ & $0.1 \pm 1.0$ \\
\hline BVRT correct & $0.3 \pm 1.5$ & $0.1 \pm 1.0$ \\
\hline BVRT errors & $0.2 \pm 1.5$ & $0.1 \pm 1.2$ \\
\hline AVLT trial 5 & $0.2 \pm 1.7$ & $0.2 \pm 1.4$ \\
\hline AVLT 30' delay & $0.3 \pm 1.6$ & $0.3 \pm 1.3$ \\
\hline CFT copy & $0.7 \pm 1.3$ & $0.3 \pm 1.4$ \\
\hline CFT recall & $0.1 \pm 1.9$ & $-0.6 \pm 1.1$ \\
\hline BNT & $0.3 \pm 1.5$ & $0.3 \pm 1.0$ \\
\hline COWA & $0.3 \pm 1.3$ & $0.0 \pm 1.3$ \\
\hline Token test & $0.6 \pm 0.2$ & $0.3 \pm 0.5$ \\
\hline Facial recognition test & $-0.1 \pm 1.2$ & $-0.3 \pm 1.1$ \\
\hline TMT A & $-0.7 \pm 3.6$ & $0.0 \pm 1.6$ \\
\hline TMT B & $-0.7 \pm 3.5$ & $-0.3 \pm 2.1$ \\
\hline Pegboard dominant & $0.0 \pm 1.4$ & $-0.9 \pm 1.9$ \\
\hline Pegboard nondominant & $0.0 \pm 0.8$ & $-0.5 \pm 1.9$ \\
\hline WCST categories & $0.0 \pm 1.1$ & $-0.4 \pm 1.7$ \\
\hline WCST perseverative errors & $-0.9 \pm 1.7$ & $-1.0 \pm 2.3$ \\
\hline & & \\
\hline
\end{tabular}

were impaired on the overall task, and again all had ratings of moderately or severely impaired adaptive function.

\section{Lesion Analysis}

To further examine the effect of lesion location on adaptive functioning, lesion maps were generated for the 15 patients with the worst adaptive functioning scores and the 15 patients with the best adaptive functioning scores

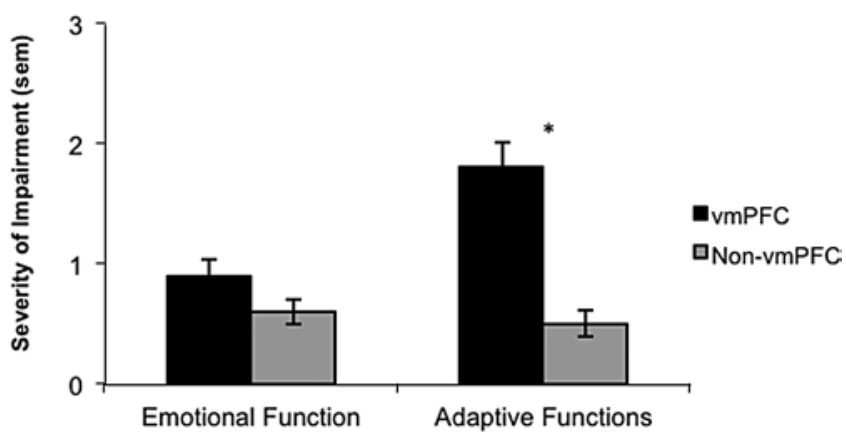

FIG. 3. Average scores in the behavioral domains for vmPFC and nonvmPFC patients. The adaptive functions score was significantly higher for the vmPFC group than for the non-vmPFC group. Numbers reflect average rating from 0 to $3(0=$ no impairment; $1=$ mild impairment; $2=$ moderate impairment; $3=$ severe impairment). ${ }^{*} p<0.001$. 

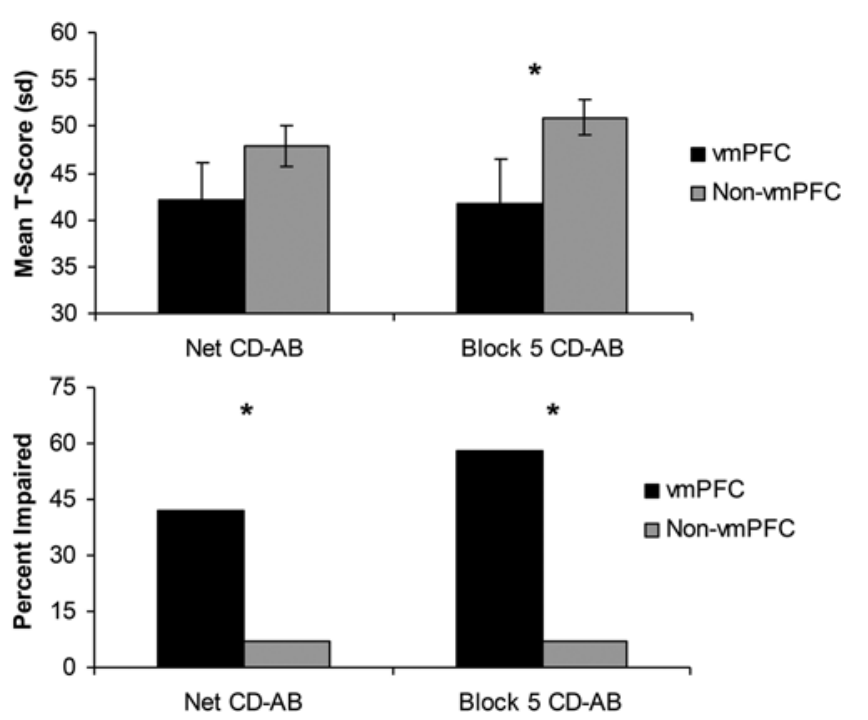

FIG. 4. Bar graphs depicting IGT performance for vmPFC and nonvmPFC groups as both a mean T-score (upper) and percent impaired (lower). ${ }^{*} p<0.05 . C D-A B=$ the number of advantageous selections made minus disadvantageous selections.

who also had available lesion data (13 patients with highest [worst] adaptive function scores and 14 patients with lowest [best] adaptive function scores; Fig. 5). Patients with the greatest deficit in adaptive functioning (upper row, Fig. 5) had a maximum lesion overlap in vmPFC (maximum overlap $=12$ ). In comparison, patients with the best adaptive functioning scores had minimal lesion overlap in any particular anatomical region (maximum overlap $=3$ ).

Next, the role of lesion size on cognitive and behavioral outcomes was examined. Regardless of whether patients were in the vmPFC or non-vmPFC groups, lesion size was correlated with impairments in attention $(\mathrm{r}=-0.38, \mathrm{p}<$ $0.003)$, memory ( $r=-0.24, p<0.05)$, verbal-language function $(\mathrm{r}=-0.29, \mathrm{p}<0.02)$, executive function $(\mathrm{r}=-0.3, \mathrm{p}<$ $0.02)$, emotional function $(r=0.23, p<0.05)$, and adaptive function $(r=0.68, p<0.001)$. To further evaluate the role of
vmPFC lesion size on adaptive function impairment, lesion size was compared for both impaired and nonimpaired patients in both the vmPFC and non-vmPFC groups (Fig. 6). When age, chronicity, and lesion size are controlled for in regression analysis, patients in the vmPFC group have significantly worse outcomes in adaptive function $(p=0.003)$ than patients in the non-vmPFC group, while patients in the non-vmPFC group have worse memory outcomes than patients in the vmPFC group $(\mathrm{p}=0.02)$. When age, chronicity, and lesion size were controlled, none of the other cognitive or behavioral domains showed significantly different levels of impairment between the vmPFC and non-vmPFC groups.

\section{Discussion}

These data demonstrate deficits in adaptive functions and real-life decision-making (measured by the IGT) in patients with meningioma-induced lesions of the vmPFC. Furthermore, we found that decline in adaptive functions from the pre- to postoperative epoch was more pronounced in patients with vmPFC lesions (compared with patients with lesions that did not involve the vmPFC). Previous work has documented associations between frontal meningiomas and personality change ${ }^{14,19}$ or neuropsychological dysfunction, ${ }^{26}$ but to our knowledge this is the first demonstration of the effects of meningioma lesions involving the vmPFC on behavioral functioning in a large group of patients. Additionally, these data demonstrate a deleterious effect of anterior skull base meningioma lesions on valuebased decision making, as measured by the IGT.

Several studies have examined neuropsychological outcomes in meningioma patients. ${ }^{8,18,26}$ Dijkstra and colleagues studied 89 patients who underwent resection of WHO Grade I meningiomas and reported significant impairment in executive functioning, verbal memory, information processing capacity, psychomotor speed, and working memory. ${ }^{8}$ In this study, patients with skull base meningiomas were more likely to have impairment of verbal memory, information processing capacity, and psy-
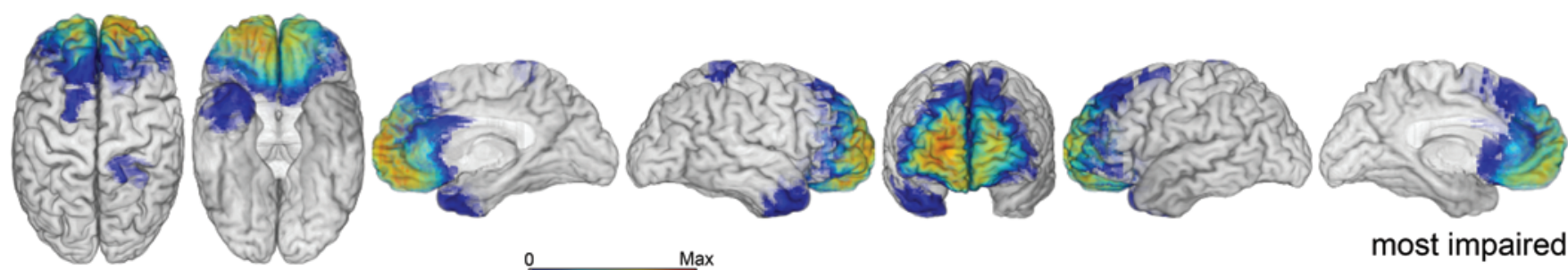

$\quad \operatorname{Max}$

most impaired
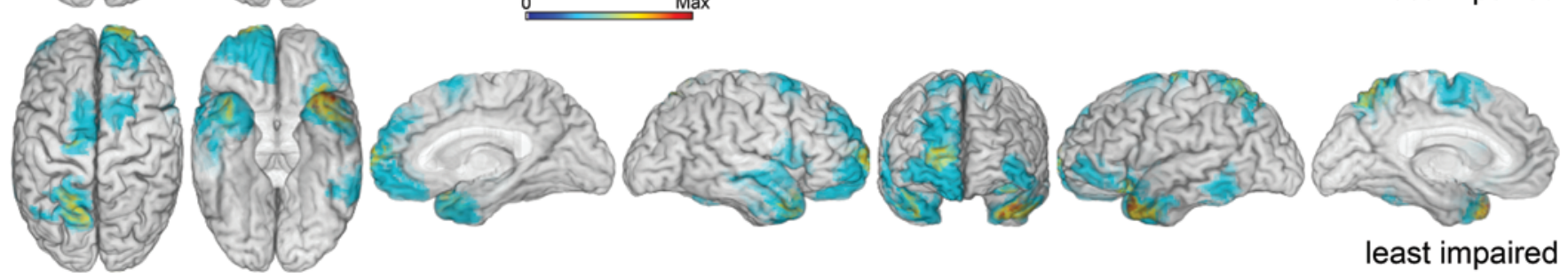

FIG. 5. Comparison of lesion overlap maps in 15 patients with greatest adaptive function impairment and least adaptive function impairment. For patients with the greatest adaptive function impairment, 14 had lesion maps (upper row; $n=14$, maximum overlap $=12$ ). For patients with the least adaptive function impairment, 13 patients had lesion overlap maps (lower row; $n=13 ;$ maximum overlap $=3$ ). These lesion overlap maps demonstrate that patients with the greatest deficits in adaptive functioning had similar lesions of the vmPFC. 


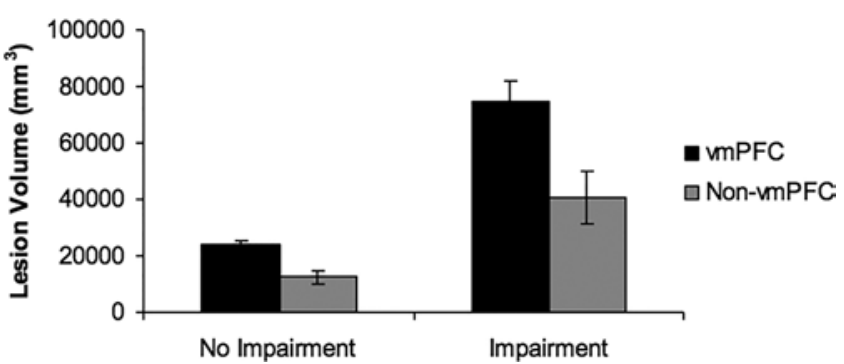

FIG. 6. Bar graph depicting lesion size as a function of adaptive function score in vmPFC and non-vmPFC lesion patients. Lesion size was associated with adaptive functioning deficits in the vmPFC group.

chomotor speed compared with convexity meningioma patients. ${ }^{8}$ Tucha et al. studied 54 patients with frontal meningiomas and found preoperative impairments in working memory, fluency functions, tonic alertness, processing speed, and flexibility, with a reported improvement in attentional functions postoperatively. ${ }^{26}$ In the present study of 70 meningioma patients, we replicated the finding of little change in cognitive function between the pre- and postoperative epochs. However, despite no significant difference between pre- and postoperative cognitive function, our patients manifested a significant decline in adaptive function postoperatively. This decline occurred in both the vmPFC and non-vmPFC groups, but was especially pronounced for patients in the vmPFC group. One interpretation for the adaptive function decline in both vmPFC and non-vmPFC groups is that neurosurgical intervention for meningioma has an effect on adaptive functions independent of meningioma location. This interpretation is consistent with previous studies showing postoperative decline in quality of life measures. ${ }^{18}$ Interestingly, for patients in the vmPFC group, greater preoperative impairment occurred in adaptive function, along with a greater decline in adaptive function between the pre- and postoperative epoch (Fig. 2). The vmPFC plays an important role in the maintenance of adaptive functions, which could help explain why vmPFC meningiomas are associated with greater preoperative adaptive function impairment. The greater magnitude of adaptive function decline postsurgically in the vmPFC group could be related to either relatively more neurosurgical trauma associated with resection of vmPFC region meningiomas (i.e., retraction injury), or that patients with preoperative adaptive function deficits have even greater difficulty coping with neurosurgical intervention, thereby leading to an even greater increase in postoperative adaptive function impairment. It is important to note that despite postoperative impairment in adaptive function, symptoms of depression (as measured by the Beck Depression Inventory) actually decreased postoperatively, and this was true in both the vmPFC and non-vmPFC groups.

In addition to the effect of vmPFC meningiomas on adaptive function, we also demonstrate deficits in valuebased decision making for patients with vmPFC meningiomas. This is not surprising given that the vmPFC plays a role in various higher cognitive functions, including valuebased decision-making ${ }^{5}$ (also moral judgments, ${ }^{17}$ emotional regulation, ${ }^{1}$ and personality ${ }^{2,3}$ ). Interestingly, we found that impairment on the IGT was associated with adaptive function impairment. Thus, it is possible that some of the adaptive function impairment occurs secondary to impaired decision making.

The mechanism of postoperative encephalomalacia (i.e., the meningioma lesion) following meningioma resection, and the pathophysiology behind the associated neuropsychological deficits, is likely a combination of local tissue ischemia (due to the meningioma itself) and neurosurgical trauma. ${ }^{12,24}$ Tomasello and colleagues documented postoperative lesion volumes in a series of patients with giant olfactory groove meningiomas and found encephalomalacia cavities smaller than the meningioma itself. ${ }^{24}$ In terms of neurosurgical trauma as a cause of postoperative encephalomalacia, approaches to resect large anterior skull base meningiomas may involve brain retraction ${ }^{24}$ or brain transgression, ${ }^{12}$ both of which could cause postoperative encephalomalacia. Meningioma growth (preoperatively) itself likely plays a substantial role in the genesis of postoperative encephalomalacia in addition to neurosurgical trauma. Using MRI, Domingo and colleagues reported decreased cerebral blood volume and increased lactate as far as $4 \mathrm{~cm}$ from meningiomas, suggesting tissue ischemia outside of the meningioma itself. ${ }^{9}$ Localized brain ischemia surrounding the meningioma may lead to cell death and concomitant encephalomalacia. Thus, meningiomas in the absence of neurosurgical intervention can cause significant disruption of surrounding brain tissue (and associated functions), and this disruption can potentially be worsened by neurosurgical trauma. Despite the effects of neurosurgical trauma and the meningioma itself, previous work has shown that cognitive functions (as measured by neuropsychological tests) typically remain stable or improve after neurosurgical resection. ${ }^{26}$ Interestingly however, our results demonstrate a marked postsurgical decline in adaptive function in meningioma patients, in the presence of stable performance on cognitive testing. This decline in adaptive functions was present in patients with both vmPFC and non-vmPFC meningioma lesions, but was especially notable in patients with vmPFC lesions.

As noted in the introduction, personality changes have been observed in many patients with frontal meningiomas. Interestingly, Krupp et al. reported that $19 \%$ of patients with meningioma did not return to their premorbid work level. It is possible that changes in the cognitive domain contribute to the alteration of personality and decreased return to work in meningioma patients, but our results suggest that it is more likely that factors within the behavioral domain (e.g., emotional function and adaptive function) are what play a key role in personality and work outcomes. We examined personality directly by measuring scores on the Iowa Scale of Personality Change as a component of emotional function (Table 3). Although we did not find significant impairment in emotional function in our patients with vmPFC lesions, there was a trend toward impairment in emotional function in patients with vmPFC lesions (Fig. 2 ). The addition of 1 or 2 patients to our study group could potentially make emotional function impairment in the vmPFC group a significant finding in our study. Our results demonstrate robust impairment in adaptive function, which is potentially related to the decreased return to work reported in other studies. ${ }^{18}$ 
Given the propensity of anterior skull base meningiomas to involve (and lesion) the vmPFC ${ }^{24}$ (e.g., gyrus rectus and orbitofrontal cortex), it is not surprising that anterior skull base meningiomas frequently present with the subjective complaint of personality change. ${ }^{21,26}$ Previously, bilateral vmPFC lesions have been associated with acquired personality change, specific deficits in dampening of emotional experience, poorly modulated emotional reactions, and defective social decision making. ${ }^{1}$

Because most meningiomas are relatively slow growing, it is possible that brain reorganization occurs in tandem with meningioma growth and that the cognitive deficits seen in meningioma patients with vmPFC lesions are less pronounced than in patients who have more abrupt lesion onset. To determine how the rate of lesion development effects neuropsychological outcome would require comparison of slow growing lesions (e.g., WHO Grade I meningiomas) to rapidly acquired lesions (e.g., a frontal contusion).

A potential limitation of this study is that our patient population is restricted to patients who underwent neuropsychological evaluation in conjunction with neurosurgical treatment. It is possible that a large number of patients with meningioma who undergo neurosurgical resection are not referred for neuropsychological evaluation. Patients referred for neuropsychological evaluation typically have more noticeable cognitive deficits or behavioral changes associated with the discovery or resection of the meningioma. For this reason, it is possible that the cohort of patients in our study has more pronounced cognitive or behavioral deficits than the typical meningioma patient. With that possibility acknowledged, our results show relative stability in the majority of cognitive functions across the pre- and postoperative epochs, consistent with other studies that had different enrollment protocols ${ }^{8,26}$ It is possible that patients referred for neuropsychological evaluation (and included in this study) are at greater risk for adaptive function impairment, but because the characteristics of pre- and postoperative cognitive change are the same in our study as in other meningioma studies, this possibility appears less likely. An additional limitation of our study is the relatively small sample size of patients who had both pre- and postoperative neuropsychological data. Although it is important to acknowledge this limitation in regard to the pre- and postoperative comparisons, the main objective of this study was to examine the effect of meningioma lesions in the vmPFC, which is well established by examination of postoperative neuropsychological tests in a large number of patients $(n=70)$ with detailed neuroanatomical lesion maps.

\section{Conclusions}

Lesions of the vmPFC resulting from meningiomas are associated with specific deficits in adaptive function and value-based decision making, despite no major dysfunction in basic cognitive abilities. Despite improvement in depression symptoms, meningioma patients with or without vmPFC involvement showed decline in adaptive function postoperatively, and this decline was especially notable in patients with vmPFC region meningiomas. Early detection and resection of meningiomas of the anterior skull base (involving gyrus rectus) may prevent these deficits.

\section{Acknowledgments}

Dr. Abel is supported by a grant through the National Institutes of Health (NIH F32-NS087664). Dr. Tranel is supported in part by a McDonnell Foundation Collaborative Award (no. 220020387).

\section{References}

1. Anderson SW, Barrash J, Bechara A, Tranel D: Impairments of emotion and real-world complex behavior following childhood- or adult-onset damage to ventromedial prefrontal cortex. J Int Neuropsychol Soc 12:224-235, 2006

2. Barrash J, Asp E, Markon K, Manzel K, Anderson SW, Tranel D: Dimensions of personality disturbance after focal brain damage: investigation with the Iowa Scales of Personality Change. J Clin Exp Neuropsychol 33:833-852, 2011

3. Barrash J, Tranel D, Anderson SW: Acquired personality disturbances associated with bilateral damage to the ventromedial prefrontal region. Dev Neuropsychol 18:355-381, 2000

4. Bechara A, Damasio H, Tranel D, Damasio AR: The Iowa Gambling Task and the somatic marker hypothesis: some questions and answers. Trends Cogn Sci 9:159-164, 2005

5. Bechara A, Tranel D, Damasio H: Characterization of the decision-making deficit of patients with ventromedial prefrontal cortex lesions. Brain 123:2189-2202, 2000

6. Damasio H, Frank R: Three-dimensional in vivo mapping of brain lesions in humans. Arch Neurol 49:137-143, 1992

7. Damasio H, Tranel D, Grabowski T, Adolphs R, Damasio A: Neural systems behind word and concept retrieval. Cognition 92:179-229, 2004

8. Dijkstra M, van Nieuwenhuizen D, Stalpers LJ, Wumkes M, Waagemans M, Vandertop WP, et al: Late neurocognitive sequelae in patients with WHO grade I meningioma. J Neurol Neurosurg Psychiatry 80:910-915, 2009

9. Domingo Z, Rowe G, Blamire AM, Cadoux-Hudson TA: Role of ischaemia in the genesis of oedema surrounding meningiomas assessed using magnetic resonance imaging and spectroscopy. Br J Neurosurg 12:414-418, 1998

10. Eslinger PJ, Damasio AR: Severe disturbance of higher cognition after bilateral frontal lobe ablation: patient EVR. Neurology 35:1731-1741, 1985

11. Frank RJ, Damasio H, Grabowski TJ: Brainvox: an interactive, multimodal visualization and analysis system for neuroanatomical imaging. Neuroimage 5:13-30, 1997

12. Gazzeri R, Galarza M, Gazzeri G: Giant olfactory groove meningioma: ophthalmological and cognitive outcome after bifrontal microsurgical approach. Acta Neurochir (Wien) 150:1117-1126, 2008

13. Gläscher J, Rudrauf D, Colom R, Paul LK, Tranel D, Damasio $\mathrm{H}$, et al: Distributed neural system for general intelligence revealed by lesion mapping. Proc Natl Acad Sci U S A 107:4705-4709, 2010

14. Hunter R, Blackwood W, Bull J: Three cases of frontal meningiomas presenting psychiatrically. BMJ 3:9-16, 1968

15. Jenkinson M, Beckmann CF, Behrens TE, Woolrich MW, Smith SM: FSL. Neuroimage 62:782-790, 2012

16. Kalkanis SN, Quiñones-Hinojosa A, Buzney E, Ribaudo HJ, Black PM: Quality of life following surgery for intracranial meningiomas at Brigham and Women's Hospital: a study of 164 patients using a modification of the functional assessment of cancer therapy-brain questionnaire. J Neurooncol 48:233-241, 2000

17. Koenigs M, Young L, Adolphs R, Tranel D, Cushman F, Hauser M, et al: Damage to the prefrontal cortex increases utilitarian moral judgements. Nature 446:908-911, 2007 
18. Krupp W, Klein C, Koschny R, Holland H, Seifert V, Meixensberger J: Assessment of neuropsychological parameters and quality of life to evaluate outcome in patients with surgically treated supratentorial meningiomas. Neurosurgery 64:40-47, 2009

19. Lampl Y, Barak Y, Achiron A, Sarova-Pinchas I: Intracranial meningiomas: correlation of peritumoral edema and psychiatric disturbances. Psychiatry Res 58:177-180, 1995

20. Lezak MD, Howieson DB, Bigler ED, Tranel D: Neuropsychological Assessment, ed 5. New York: Oxford University Press, 2012

21. Nakazato Y, Hirato J: A 29-year-old woman who presented with personality change and a tumor in the frontal skull base. Neuropathology 25:395-397, 2005

22. Okhovat A: A 46-year-old woman with a history of personality change, headache, and olfactory hallucination. Iran J Neurol 12:77-79, 2013

23. Pizzoni C, Sarandria C, Pierangeli E: Clear-cell meningioma of the anterior cranial fossa. Case report and review of the literature. J Neurosurg Sci 53:113-117, 2009

24. Tomasello F, Angileri FF, Grasso G, Granata F, De Ponte FS, Alafaci C: Giant olfactory groove meningiomas: extent of frontal lobes damage and long-term outcome after the pterional approach. World Neurosurg 76:311-317, 255-258, 2011

25. Tranel D, Hathaway-Nepple J, Anderson SW: Impaired behavior on real-world tasks following damage to the ventromedial prefrontal cortex. J Clin Exp Neuropsychol 29:319-332, 2007

26. Tucha O, Smely C, Preier M, Becker G, Paul GM, Lange $\mathrm{KW}$ : Preoperative and postoperative cognitive functioning in patients with frontal meningiomas. J Neurosurg 98:21-31, 2003

\section{Disclosures}

The authors report no conflict of interest concerning the materials or methods used in this study or the findings specified in this paper.

\section{Author Contributions}

Conception and design: Abel, Belfi, Tranel. Acquisition of data: Abel, Manzel, Bruss, Tranel. Analysis and interpretation of data: Abel, Manzel, Tranel. Drafting the article: Abel. Critically revising the article: all authors. Reviewed submitted version of manuscript: all authors. Approved the final version of the manuscript on behalf of all authors: Abel. Statistical analysis: Manzel, Bruss. Study supervision: Tranel.

\section{Supplemental Information}

\section{Previous Presentations}

An abstract was accepted and presented as a poster at the 2015 AANS annual meeting (Washington, DC, May 2-6) containing portions of the data presented in this manuscript.

\section{Correspondence}

Taylor J. Abel, Department of Neurosurgery, University of Iowa, 200 Hawkins Dr., Iowa City, IA 52242. email: taylor-abel@ uiowa.edu. 